\section{P195 CREATION OF AN HIV CASE DEFINITION TO ASSESS THE QUALITY OF HIV CARE IN THE PRIMARY HEALTHCARE SETTING IN CANADA}

${ }^{1}$ Shabnam Asghari, ${ }^{1}$ Sarah Boyd*, ${ }^{2}$ jillian Blackmore, ${ }^{3}$ Tao Chen, ${ }^{4}$ Marissa Becker, ${ }^{5}$ Claire Kendall, ${ }^{6}$ Alexander Singer. ${ }^{1}$ Memorial University Centre for Rural Health Studies, St. John's, Canada; ${ }^{2}$ Former Research Assistant at Memorial University Centre for Rural Health Studies, St. John's, Canada; ${ }^{3}$ Canadian Primary Care Sentinel Surveillance Network, Kingston, Canada; ${ }^{4}$ University of Manitoba, Centre for Global Public Health, Department Of Community Health Sciences, Winnipeg, Canada; ${ }^{5}$ University of Ottawa, Ottawa, Canada; ${ }^{6}$ University of Manitoba, Winnipeg, Canada

\subsection{6/sextrans-2019-sti.346}

Background People living with the human immunodeficiency virus (PLHIV) have ongoing healthcare needs as HIV has become a chronic condition for those in treatment. With the success of antiretroviral (ARV) medications, AIDS-related illnesses are no longer the biggest threat to PLHIV, rather, emerging complications and ARV toxicities are of concern. For this reason, HIV care is now transitioning to primary care. To be able to assess the quality of HIV care in these settings a valid case definition is required. Our objective was to develop and validate a case definition for HIV applicable to the Canadian Primary Care Sentinel Surveillance Network (CPCSSN) database.

Methods Electronic Medical Record data from CPCSSN (exclusively primary care data) was used to develop a retrospective cohort between 2009 and 2016. We identified all possible records of $\mathrm{HIV}$ in the dataset based on the presence of HIV codes, keywords and ARVs. Every combination of codes, keywords and ARVs were analyzed to see which resulted in the most accurate definition of PLHIV. To assess the validity, we linked the data to a LHIV cohort (external reference standard) in Newfoundland and Labrador; and, a random sample of the CPCSSN database which was reviewed by two experts to confirm HIV status (internal reference standard). Sensitivity, specificity and predictive values were measured.

Results It was determined that the presence of an HIV keyword in the EMR along with either an ICD code or taking 3 or more ARV drugs was the most accurate algorithm for predicting PLHIV. Compared to internal and external references, the algorithm showed $(97.1 \%$ and $95.0 \%$ sensitivity, $100 \%$ and $80 \%$ specificity), respectively.

Conclusion This is the first Canada-wide study investigating the utilization of primary healthcare by PLHIV. This case definition will contribute to future research and improvements in providing care to PLHIV in a primary care setting.

Disclosure No significant relationships.

\section{P196 WIR-CENTER FOR SEXUAL HEALTH AND MEDICINE: A CARE MODEL TO COUNTER HIVISTI IN GERMANY}

\footnotetext{
${ }^{1}$ Norbert Brockmeyer*, ${ }^{1}$ Christoph Schlüter, ${ }^{1}$ Anja Potthoff, ${ }^{2}$ Janet Wach, ${ }^{3}$ Arne Kayser, ${ }^{4}$ Martina Schu, ${ }^{5}$ Benjamin Jonas, ${ }^{5}$ Peter Tossmann, ${ }^{1}$ Andre Kasper, ${ }^{6}$ Adriane SkaletzRorowski. 'Walk In Ruhr - WIR - Center for Sexual Health, Dept. of Dermatology, Ruhruniversität Bochum, Bochum, Germany; '2Public Health Department Bochum, Bochum, Germany; ${ }^{3}$ AIDS Service Organization Bochum e.V., Bochum, Germany; ${ }^{4}$ FOGS GmbH, Cologne, Germany; ${ }^{5}$ Delphi Gesellschaft für Forschung, Beratung und Projektentwicklung mbH, Berlin, Germany; ${ }^{6}$ Walk In Ruhr - WIR - Center for Sexual Health, Dept. of Dermatology, Ruhr-universität Bochum, Bochum, Germany
}

10.1136/sextrans-2019-sti.347
Background Increasing incidence of sexually transmitted infections (STI) in Germany has shown the necessity of easily accessible sexual health services. Walk In Ruhr (WIR), a unique inter-institutional Center for Sexual Health and Medicine in Germany, unites the Outpatient Clinic, Public Health Department and four community-driven NGO. Together, they provide counseling, testing and treatment for different target groups (e.g. youth, MSM, swinger) in one building. Innovative methods in preventing HIV/STI [Online Partner Notification tool (PN), PrEP, Online Risk Test (ORT), HIV/STI-self-sampling-kit 'teST-It'] are developed and tested here. Health Advisers offer counseling, outreach-work and guidance to specialists.

Methods During a 21-month-evaluation-period, supported by the German Ministry of Health, clients completed a quantitative survey. Additional surveys, conducted independently by the WIR for PrEP- and 'teST-It'- users were included, the ORT, the PN usage and HIV/STI test diagnosis and treatment were analyzed.

Results The evaluation is ongoing. Until 2/2019 approx. 3.200 surveys will be completed. Initial results show that target groups differ regarding age, gender, sex-orientation, visit reason and WIR institutions they approach. Additional studies show that a quarter of the clients are HIV-positive. $10.5 \%$ of the clinic's patients and 10\% of the Public Health Dept. clients were tested STI-positive. Treatment of C. trachomatis and N. gonorrhoeae was successful in 98\% of all cases and in 93.5\% of all M. genitalium cases. PrEP usage has been rising (144 new PrEP users since 10/2017) and the PN tool has been used 208 times since 06/2017. Since 12/2017, 4502 persons used the anonymous ORT. 'teST-It' is available since 09/ 2018.

Conclusion In contrast to common care concepts, WIR achieves high levels of recognition and acceptance among different target groups and the average population. The opportunity to access different organizations helps to meet the medical and counseling needs of the clients to reduce further transmission of HIV/STI.

Disclosure No significant relationships.

\section{P198 DOLUTEGRAVIR ROLLOUT AND EXPECTED PREVALENCE OF PRETREATMENT DRUG RESISTANCE TO ANTIRETROVIRAL THERAPY AMONG KENYAN WOMEN}

${ }^{1}$ Horacio Duarte* ${ }^{*}{ }^{2}$ Eva Enns, ${ }^{3}$ David Stauffer, ${ }^{4}$ Robert Shafer, ${ }^{4}$ Eran Bendavid. ${ }^{1}$ University of Washington School of Medicine, Pediatric Infectious Diseases, Seattle, USA; ${ }^{2}$ University of Minnesota School of Public Health, Minneapolis, USA; ${ }^{3}$ University of Washington School of Medicine, Sunnyvale, USA; ${ }^{4}$ Stanford University School of Medicine, Stanford, USA

\subsection{6/sextrans-2019-sti.348}

Background The prevalence of pretreatment HIV drug resistance (PDR) to efavirenz-based ART is increasing in East Africa, which may decrease the effectiveness of antiretroviral therapy (ART) programs. The recent implementation of dolutegravir-based ART is an important strategy to address PDR. However, concerns about a potential increased risk of neural tube defects associated with use of dolutegravir by women at the time of conception will likely prevent a large proportion of women from using this drug.

Methods We developed an HIV simulation model to project the prevalence of PDR among Kenyan women prior to initiating ART, as well as to evaluate multiple health outcomes 
among men and women, including rate of virologic failure, over a 15-year time horizon, starting in 2019. The model simulated the emergence and transmission of resistance mutations to efavirenz-based ART, calibrated to the Kenyan epidemic. Our base-case scenario assumed dolutegravir coverage gradually increased such that, by 2022, $100 \%$ of men initiating ART receive dolutegravir and only $40 \%$ of women initiating ART receive dolutegravir.

Results In 2019, the baseline PDR prevalence among women was $11.5 \%$. In the base-case scenario, PDR prevalence peaked in 2021 to $12.3 \%$ and by the end of 2034 was $8.6 \%$. On average over 15 years, among patients with PDR to efavirenz, $61.7 \%$ of men and $59.2 \%$ of women achieved viral suppression. When we assumed 100\% dolutegravir coverage for women by 2022, PDR prevalence among women was $7.8 \%$ by the end of 2034 .

Conclusion Although efavirenz-associated PDR prevalence is projected to decrease over time as dolutegravir coverage expands, the prevalence of PDR will remain high among women, even several years from now. It remains important to identify cost-effective strategies to address PDR in populations for whom dolutegravir is not an option, particularly women.

Disclosure No significant relationships.

\section{\begin{tabular}{|l|l}
\hline P199 TRENDS IN MOTIVATION AND SETTING FOR HIV \\
\hline
\end{tabular} TESTING AMONG PEOPLE NEWLY DIAGNOSED WITH HIV IN BRITISH COLUMBIA, CANADA, 2003-2007}

\begin{abstract}
${ }^{1}$ Theodora Consolacion, ${ }^{2}$ Mark Hull, ${ }^{3}$ Robin Yates, ${ }^{1}$ Monica Durigon, ${ }^{1}$ Geoffrey Ford,

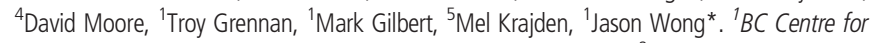
Disease Control, Clinical Prevention Services, Vancouver, Canada; ${ }^{2}$ British Columbia Centre for Excellence in HIVIAIDS, Vancouver, Canada; ${ }^{3} B C$ Ministry of Health, Victoria, Canada; ${ }^{4} B C$ Centre for Excellence in HIV/AIDS, Vancouver, Canada; ${ }^{5} B C$ Centre for Disease Control Public Health Laboratory, Vancouver, Canada
\end{abstract}

\subsection{6/sextrans-2019-sti.349}

Background In British Columbia (BC), Canada, new HIV testing guidelines introduced in 2014 recommended routine offer of HIV testing in an effort to diagnose HIV earlier. We assessed changes in motivation and setting for HIV testing that resulted in a new diagnosis of HIV to evaluate this strategy.

Methods In 2013, questions about who initiated testing (provider, client, or other/unknown), test setting (healthcare, community [e.g. outreach, peer], or other), and reason for testing (e.g. recent risk event, routine test, diagnosed with another sexually transmitted or bloodborne infection [STIBBI]) were added to the HIV case report form. Trends from 2003 to 2017 were assessed using Cochran-Armitage tests. HIV testing volumes were determined from the provincial laboratory, which performs $>95 \%$ of all HIV tests in BC.

Results HIV testing increased from 223,300 episodes in 2013 to 337,900 in 2017. New diagnoses of HIV decreased from 265 in 2013 to 186 in 2017 (cumulative total 1,193). An increasing trend was noted for reporting testing in a healthcare setting compared to community or other settings $(\mathrm{p}<0.01)$, especially among men who have sex with men $(\mathrm{p}<0.01)$ and people who inject drugs $(\mathrm{p}<0.01)$. Provider initiated testing was reported by half of all new HIV diagnoses and remained stable $(p=0.86)$. There was a decreasing trend for reporting recent risk event/exposure $(p=0.03)$ and being notified as a contact $(p=0.01)$ as the reason for testing, and an increasing trend for reporting being diagnosed with another STIBBI $(p=0.03)$. There was no trend in reporting the reason for testing as routine test $(p=0.74)$.

Conclusion Routine offer of HIV testing may be increasing the proportion of HIV diagnosed in healthcare settings. The increase in proportion of new HIV diagnoses due to diagnosis of another STIBBI highlights the importance of co-testing of HIV and other STIBBIs.

Disclosure No significant relationships.

\section{P202 DEVELOPING A DATABASE WITH SENSITIVE HEALTH INFORMATION: A PROFILE OF PEOPLE LIVING WITH HIV IN NEWFOUNDLAND AND LABRADOR}

${ }^{1}$ Shabnam Asghari, 'Sarah Boyd*, ${ }^{2}$ Jillian Blackmore, ${ }^{1}$ Oliver Hurley, ${ }^{3}$ John Knight, ${ }^{4}$ Deborah Kelly, ${ }^{5}$ Kimberly Burt, ${ }^{3}$ Beatrice Pittman, ${ }^{6}$ Laura Gilbert, ${ }^{3}$ Jeff Dowden. ${ }^{1}$ Memorial University Centre for Rural Health Studies, St. John's, Canada; ${ }^{2}$ Former Research Assistant at Memorial University Centre for Rural Health Studies, St. John's, Canada; ${ }^{3}$ Newfoundland and Labrador Centre for Health Information, St. John's, Canada; ${ }^{4}$ Memorial University School of Pharmacy, St. John's, Canada; ${ }^{5}$ Eastern Health, St. John's, Canada; ${ }^{6}$ Newfoundland and Labrador Public Health Laboratory, St. John's, Canada

\subsection{6/sextrans-2019-sti.350}

Background In Newfoundland and Labrador (NL), people living with HIV (PLHIV) primarily receive care through an interdisciplinary HIV specialty clinic, however, HIV care has been transitioning to primary healthcare elsewhere. Developing a comprehensive cohort of PLHIV to help improve healthcare has long been the vision of researchers, clinicians and decision makers. The objective of this study was to develop a de-identified cohort of PLHIV in NL, to be used to inform policy and prioritize healthcare system changes to optimize HIV healthcare in NL and to address gaps in care for PLHIV.

Methods Data was collected from a number of different databases and PLHIV were identified as having HIV from three sources: 1. NL Public Health Laboratory data; 2. HIV Clinic data; 3. Medical Administration data.

Results The finalized dataset included 317 people who had been diagnosed with HIV in NL as of 1994 and 251 were still alive at the end of data collection. The final database contained a total of 178 variables describing PLHIV health and health care utilization. Only $7 \%$ of PLHIV were identified by all three sources suggesting no single provincial HIV data custodian captures all those living with HIV in the province. Conclusion It is important that policy be implemented to merge siloed data sources in order to provide researchers, clinicians and decision makers with the accurate and complete data that is required to conduct sound and precise research, provide appropriate care and allocate resources to health initiatives that can improve the quality of life for PLHIV.

Disclosure No significant relationships. 